\title{
Numerical Analysis of the Exterior Boundary Value Problem for the Time-Harmonic Maxwell Equations by a Boundary Finite Element Method Part 1: The Continuous Problem
}

\author{
By A. Bendali
}

\begin{abstract}
A general finite element method is applied to compute the skin currents flowing on a perfectly conducting surface when it is illuminated by a time-harmonic incident electromagnetic wave. In this paper, we introduce and study the framework in which the continuous problem can be stated in order to make possible the numerical analysis which will follow in a second part.
\end{abstract}

0. Introduction. The determination of the diffracted field by a perfectly conducting obstacle $\Gamma$ (which is supposed here to be the smooth boundary of a bounded open domain $\Omega^{i}$ ) is reduced to that of the surface currents $\vec{j}$ and charges $\rho$ on $\Gamma$ (cf. e.g. [11], [15], [22]) which satisfy the integral equation

$$
\Pi\left\{-\frac{1}{\varepsilon} \operatorname{grad} v+i \omega \mu \vec{a}\right\}=-\Pi \vec{e}^{\mathrm{inc}} \text { on } \Gamma .
$$

$\Pi$ is the orthogonal projection on the tangent plane of $\Gamma ; \vec{e}^{\mathrm{inc}}$ is the electric part of the incident electromagnetic wave; $\varepsilon$ and $\mu$ are the characteristic constants of the medium in which $\Gamma$ is embedded. The time variation is supposed to be $e^{-i \omega t}$ and is suppressed by linearity; $v$ and $\vec{a}$ are respectively the scalar and the vector potential of the electric field diffracted by $\Gamma$, respectively created by the surface charges $\rho$ and the surface currents $\vec{j}$ :

$$
\begin{gathered}
v(x)=\int_{\Gamma} G(x, y) \rho(y) d \gamma(y), \\
\vec{a}(x)=\int_{\Gamma} G(x, y) \vec{j}(y) d \gamma(y) . \\
G(x, y)=\frac{e^{i k|x-y|}}{4 \pi|x-y|}
\end{gathered}
$$

is the kernel giving the outgoing solutions of the Helmholtz equation;

$$
k=\omega \sqrt{\varepsilon \mu}
$$

is the wave number. 
Finally, the currents $\vec{j}$ and the charges $\rho$ induced by the incident field $\vec{e}^{\mathrm{inc}}$ on $\Gamma$ are linked by the conservation law

$$
\operatorname{div}_{\Gamma} \vec{j}=i \omega \rho,
$$

where $\operatorname{div}_{\Gamma} \vec{j}$ is the surface divergence of the tangential field $\vec{j}$ of $\Gamma$.

Rumsey [19] proposed the concept of reaction between two systems of electromagnetic sources in order to replace Eq. $(0.1)$ by the following variational equation

$$
\int_{\Gamma}\left(-\frac{1}{\varepsilon} \operatorname{grad} v+i \omega \mu \vec{a}, \vec{q}\right) d \gamma=-\int_{\Gamma}\left(\vec{e}^{\mathrm{inc}}, \vec{q}\right) d \gamma
$$

for all tangential fields $\vec{q}$. In the sequel, $(\cdot, \cdot)$ will denote the Hermitian product of two vectors with three complex components.

Sankar and Tong [20] used the formulation (0.7) together with the gauge relation

$$
\operatorname{div} \vec{a}=i \omega v
$$

to treat the case where $\Gamma$ is a metallic plate. An important remark about this formulation is that it is not difficult to deal with the case of open surfaces. It is sufficient to impose on the unknown currents $\vec{j}$ and on the test currents $\vec{q}$ not to have any normal component to the boundary curve $\partial \Gamma$ of $\Gamma$. This is not the case with the magnetic integral equation also called the Maue equation; cf. e.g. [22]. However, the method proposed by Sankar and Tong is too restrictive since it cannot take into account arbitrarily shaped surfaces. Moreover, they do not elucidate the way the singularity $1 /|x-y|^{3}$, which appears in this formulation, is numerically handled.

Harrington and Mautz, in a series of papers (cf. [9]) proposed to carry out the integration by parts

$$
\int_{\Gamma}(\operatorname{grad} v, \vec{q}) d \gamma=\int_{\Gamma}\left(\operatorname{grad}_{\Gamma} v, \vec{q}\right) d \gamma=-\int_{\Gamma} v \overline{\operatorname{div}_{\Gamma} \vec{q}} d \gamma
$$

where $\operatorname{grad}_{\Gamma}$ is the surface gradient of a function defined on $\Gamma$. Equation (0.7) together with the conservation law (0.6) leads then to the problem

$$
\left\{\begin{array}{l}
\text { Find a tangential field } \vec{j} \text { on } \Gamma \text { such that, for all tangential fields } \vec{q} \text { of } \Gamma \\
\int_{\Gamma \times \Gamma} G(x, y)\left\{-\frac{1}{k^{2}} \operatorname{div}_{\Gamma} \vec{j}(y) \overline{\operatorname{div}_{\Gamma} \vec{q}(x)}+(\vec{j}(y), \vec{q}(x))\right\} d \gamma(x) d \gamma(y) \\
\quad=-\frac{1}{i \omega \mu} \int_{\Gamma}\left(\vec{e}^{\mathrm{inc}}, \vec{q}\right) d \gamma
\end{array}\right.
$$

An important feature of the above problem must be pointed out: in the equation, there appears only the kernel $G$ and not its derivatives. Thus the integrals remain weakly singular.

It seems that Harrington and Mautz were faced with the construction of a conforming finite element space of currents on $\Gamma$ (in physical terms a space for which there do not exist line or vertex charges). This is the reason why, as far as we know, they only treat bidimensional and axisymmetric problems by the formulation (0.10).

Recently, Rao et. al [16] proposed the following method. The surface is replaced by an approximate polyhedral surface $\Gamma_{h}$ formed by a juxtaposition of planar triangles. The currents and the charges are then determined by the mixed finite 
element method of the lowest order, where the currents flowing across any edge and, consequently, the charges over any triangle are supposed to be constant. This method was actually introduced by Raviart and Thomas [17] for solving the mixed formulation of the Poisson equation in a domain in the plane. It was next used by Nedelec [13] for the computation of eddy currents on a surface of $\mathbf{R}^{3}$. As shown in [2], Rao's method can be generalized, in light of the work of Raviart and Thomas, to a method of arbitrary order, if $\Gamma$ is a polyhedral. However, in the case where $\Gamma$ is a curved surface, if the directions of the tangent plane are not correctly approximated (i.e. if they are only approximated by the directions of the plane triangle), we have a loss of one convergence order. A similar problem arises in double layer potential formulations which require the interpolation of the normal (cf. [18]).

However, as far as we know, up to now nobody has treated the numerical analysis of the problem (i.e. the existence and uniqueness of the solution of the approximate problem and error estimates.) This will be the subject of this work, divided into two parts. In this first part, we state the boundary value problem which leads to Eq. (0.1). We recall the existence and uniqueness results for this problem (cf. [1]) and give the regularity properties of its solution. This enables us to state the variational equation (0.10) in a suitable framework. Finally, we give some coerciveness estimates on which the numerical analysis developed in the second part of this work will be based.

There exist other methods of discretization of the problem of scattering an electromagnetic wave by a perfectly conducting obstacle. A comprehensive account of these methods and relevant references may be found in [15] and [16]. It must be emphasized that the list of references given above is by no means complete. We only tried to illustrate some of the important features of the method which will be discussed.

\section{Review of Some Facts About Boundary Value Problems.}

1.1. Notation and Spaces. $\Omega^{i}$ is a bounded open subset of $\mathbf{R}^{3}$. Its boundary $\Gamma$ is a smooth (i.e. $C^{\infty}$ ) surface of $\mathbf{R}^{3}$. $\Omega^{i}$ lies locally on one side of $\Gamma$ and is such that the exterior domain $\Omega^{e}=\mathbf{R}^{3} \backslash \bar{\Omega}^{i}$ is connected. We suppose that $\Omega^{i}$ is also connected (the case where $\Omega^{i}$ is not connected differs only by some inessential details in the proofs). Hence, $\Gamma$ is also the boundary of $\Omega^{e}$ and is connected. We shall denote by $\vec{n}$ the field formed by the unit normal to $\Gamma$ outwardly directed to $\Omega^{i}$. One can see (cf. e.g. [4]) that the field $\vec{n}$ can be extended to $\mathbf{R}^{3}$ to define a $C^{\infty}$ field, also called $\vec{n}$, and compactly supported, such that $|\vec{n}| \equiv 1$ in a neighborhood of $\Gamma$. In the sequel, $\Omega$ represents either $\Omega^{i}$ or $\Omega^{e}$ for any purpose which does not need the boundedness of the domain; $\vec{n}$ is then the unit normal to $\Gamma$ outwardly directed to $\Omega$.

For $s \in \mathbf{R}$, we denote by $\mathbf{H}^{s}(\Omega)$ (resp. $\left.\mathbf{H}^{s}(\Gamma)\right)$ the space formed by the fields $\vec{u}$ with complex components which belong to $H^{s}(\Omega)$ (resp. $H^{s}(\Gamma)$ ). $\mathbf{H}^{s}(\Omega)$ (resp. $\left.\mathbf{H}^{s}(\Gamma)\right)$ is identified, for any fixed orthonormal basis $\left\{\vec{e}_{1}, \vec{e}_{2}, \vec{e}_{3}\right\}$ to $\left\{H^{s}(\Omega)\right\}^{3}$ (resp. $\left.\left\{H^{s}(\Gamma)\right\}^{3}\right)$, by

$$
\vec{u}=u^{i} \vec{e}_{i} ; \quad u^{i} \in H^{s}(\Omega)\left(\operatorname{resp} . H^{s}(\Gamma)\right) .
$$

We shall always take the usual convention of tensorial calculus of summing over the repeated indices. As a rule, latin indices go from 1 to 3, while greek ones take the values 1 or 2 . 
$H^{s}(\Omega)\left(\right.$ resp. $\left.H^{s}(\Gamma)\right)$ denotes the usual Sobolev space (cf. [10]). For $\vec{u}$ in $\mathbf{H}^{s}(\Omega)$, by the decomposition (1.1), we can use the norm notation

$$
\|\vec{u}\|_{s, \Omega}=\left(\sum_{i=1}^{3}\left\|u^{i}\right\|_{s, \Omega}^{2}\right)^{1 / 2}
$$

and similar notations for $\|\vec{u}\|_{s, \Gamma}$ and for the seminorm $|\vec{u}|_{m, K}$, if $m$ is a nonnegative integer and $K$ a measurable subset of $\Omega$ or $\Gamma .\left\|u^{i}\right\|_{s, \Omega}$ is the usual norm of $u^{i}$ in $H^{s}(\Omega)$.

The inner product of two vectors $\vec{a}=a^{i} \vec{e}_{i}$ and $\vec{b}=b^{i} \vec{e}_{i}$ is denoted by

$$
(\vec{a}, \vec{b})=\sum_{i=1}^{3} a^{i} \bar{b}^{i}
$$

$\vec{a} \wedge \vec{b}$ denotes the usual vectorial product.

Using the stability of Sobolev spaces with respect to the multiplication by $C^{\infty}$ functions, we can decompose $\mathbf{H}^{s}(\Gamma)$ into two subspaces respectively generated by the tangential fields to $\Gamma$ and the normal fields to $\Gamma$. We have

$$
\begin{aligned}
\vec{p} & =\Pi \vec{p}+(\vec{p}, \vec{n}) \vec{n} ; \\
N H^{s}(\Gamma) & =\left\{\vec{p} \in \mathbf{H}^{s}(\Gamma): \vec{p}=v \vec{n} ; v \in H^{s}(\Gamma)\right\} ; \\
T H^{s}(\Gamma) & =\left\{\vec{p} \in \mathbf{H}^{s}(\Gamma):(\vec{p}, \vec{n})=0\right\} ; \\
\mathbf{H}^{s}(\Gamma) & =T H^{s}(\Gamma) \oplus N H^{s}(\Gamma) .
\end{aligned}
$$

This last direct sum is also an orthogonal decomposition if $\mathbf{H}^{s}(\Gamma)$ is equipped with a suitable norm.

If $X$ is a Hilbert space, $X^{\prime}$ denotes the space of all antilinear bounded forms on $X$. $\langle l, v\rangle_{X^{\prime} . X}$ denotes the duality pairing between $X$ and $X^{\prime}$. Nevertheless, to simplify the notations, we shall omit $X$ and $X^{\prime}$ if there is no risk of confusion.

Finally, the usual identifications of duality of Sobolev spaces enable us to take

$$
\left\{T H^{s}(\Gamma)\right\}^{\prime}=T H^{-s}(\Gamma) ; \quad\left\{N H^{s}(\Gamma)\right\}^{\prime}=N H^{-s}(\Gamma) .
$$

We shall use the following Fréchet spaces (cf. [23]):

$$
\begin{aligned}
\mathbf{H}_{\mathrm{loc}}^{s}\left(\bar{\Omega}^{e}\right) & =\left\{\vec{u} \in\left\{\mathscr{D}^{\prime}\left(\bar{\Omega}^{e}\right)\right\}^{3}: \varphi \vec{u} \in \mathbf{H}^{s}\left(\Omega^{e}\right) \forall \varphi \in \mathscr{D}\left(\mathbf{R}^{3}\right)\right\}, \\
\mathbf{H}_{\mathrm{loc}}^{1}\left(\Delta, \bar{\Omega}^{e}\right) & =\left\{\vec{u} \in \mathbf{H}_{\mathrm{loc}}^{1}\left(\bar{\Omega}^{e}\right): \Delta \vec{u} \in \mathbf{L}_{\mathrm{loc}}^{2}\left(\Omega^{e}\right)\right\} .
\end{aligned}
$$

Recall that $\Delta \vec{u}=\operatorname{grad} \operatorname{div} \vec{u}-\operatorname{curl}$ curl $\vec{u}$ is given by $\Delta \vec{u}=\left(\Delta u^{i}\right) \vec{e}_{i}$ when $\vec{u}$ is in the form (1.1). $\mathbf{H}^{1}(\Delta, \Omega)$ being defined in a similar way, one can follow [10] to define, for $\vec{u}$ in $\mathbf{H}_{\mathrm{loc}}^{1}(\Delta, \bar{\Omega})$, the traces curl $\vec{u} \wedge \vec{n}$ and $\gamma_{0}(\operatorname{div} \vec{u})$, respectively, in $T H^{-1 / 2}(\Gamma)$ and $H^{-1 / 2}(\Gamma)$. This definition uses the surjectivity, the trace operator from $H^{1}(\Omega)$ onto $\mathbf{H}^{1 / 2}(\Gamma)$, the density of the space of smooth functions in $\mathbf{H}^{1}(\Delta, \Omega)$ and the following Green's formula which holds for sufficiently smooth $\vec{u}$ and $\vec{v}$ :

$$
\begin{aligned}
& \int_{\Omega}\{(\Delta \vec{u}, \vec{v})+(\operatorname{curl} \vec{u}, \operatorname{curl} \vec{v})+\operatorname{div} \vec{u} \overline{\operatorname{div} \vec{v}}\} d x \\
& =\int_{\Gamma}\left\{(\operatorname{curl} \vec{u} \wedge \vec{n}, \vec{v})+(\vec{n}, \vec{v}) \gamma_{0} \operatorname{div} \vec{u}\right\} d \gamma
\end{aligned}
$$


It can easily be seen that this definition of $\gamma_{0}(\operatorname{div} \vec{u})$ coincides with the one given by Lions and Magenes [10], which is based on Green's formula:

$$
-\langle\Delta(\operatorname{div} \psi \vec{u}), \varphi\rangle_{H^{-1}(\Omega), H_{0}^{1}(\Omega)}+\int_{\Omega} \operatorname{div}(\psi \vec{u}) \overline{\Delta \varphi} d x=\left\langle\gamma_{0} \operatorname{div} \vec{u}, \gamma_{1} \varphi\right\rangle
$$

where $\psi$ is any function of $\mathscr{D}\left(\mathbf{R}^{3}\right), \psi \equiv 1$ near $\Gamma$ and $\gamma_{1} \varphi$ is the second trace $\partial \varphi / \partial \vec{n}$ of $\varphi \in H^{2}(\Omega) \cap H_{0}^{1}(\Omega)$. In the same way, the definition of curl $\vec{u} \wedge \vec{n}$, by (1.9), coincides with the one given by Duvaut and Lions [5] if curl curl $\vec{u}$ belongs to $\mathbf{L}_{\text {loc }}^{2}(\Omega)$.

1.2. The Boundary Value Problems. Let a tangential field $\vec{c} \in T H^{1 / 2}(\Gamma)$ and $g \in H^{-1 / 2}(\Gamma)$ be given. For a given positive real number $k$, it has previously been shown (cf. [1]), that the exterior problem

$$
\begin{cases}\text { Find } \vec{e} \in \mathbf{H}_{\mathrm{loc}}^{1}\left(\Delta, \bar{\Omega}^{e}\right) \text { such that } \\ \Delta \vec{e}+k^{2} \vec{e}=0 & \text { in } \Omega^{e} \\ \Pi \vec{e}=\vec{c} & \text { in } T H^{1 / 2}(\Gamma), \\ \gamma_{0} \operatorname{div} \vec{e}=g & \text { in } H^{-1 / 2}(\Gamma), \\ \operatorname{curl} \vec{e} \wedge \vec{r} / r+(\vec{r} / r) \operatorname{div} \vec{e}-i k \vec{e}=o(1 / r),\end{cases}
$$

has one and only one solution. We have used the following notations: $\vec{r}$ is the radius vector of the generic point $x$ in $\mathbf{R}^{3}, r$ its length and $o(1 / r)$, as usual, denotes a function going to zero faster than $1 / r$ when $r$ tends to infinity uniformly with respect to the angular directions $\vec{r} / r$.

In [1], it has been proved also that, if $k^{2}$ is not an eigenvalue of the interior problem (in the sequel, we shall always assume this hypothesis), the problem

$$
\begin{cases}\text { Find } \vec{e} \in \mathbf{H}^{1}\left(\Delta, \Omega^{i}\right) \\ \Delta \vec{e}+k^{2} \vec{e}=0 & \text { in } \Omega^{i} \\ \Pi \vec{e}=\vec{c} & \text { in } T H^{1 / 2}(\Gamma) \\ \gamma_{0} \operatorname{div} \vec{e}=g & \text { in } H^{-1 / 2}(\Gamma)\end{cases}
$$

has one and only one solution. Moreover, in this case, it is known that $k^{2}$ is not an eigenvalue of the interior Dirichlet problem for the Laplace equation.

The regularity properties of the solution of (1.11) and (1.12) are given by

THEOREM 1.1. Let $\vec{u}$ in $\mathbf{H}_{\mathrm{loc}}^{1}(\Delta, \bar{\Omega})$ be such that, for $s \geqslant 0$,

$$
\begin{aligned}
& \Delta \vec{u} \in \mathbf{H}_{\mathrm{loc}}^{t}(\bar{\Omega}), \quad t=\max (0, s-1), \\
& \Pi \vec{u} \in T H^{s+1 / 2}(\Gamma) \\
& \gamma_{0} \operatorname{div} \vec{u} \in H^{s-1 / 2}(\Gamma)
\end{aligned}
$$

Then,

$$
\vec{u} \in \mathbf{H}_{\mathrm{loc}}^{s+1}(\bar{\Omega}) .
$$


Proof. $\vec{n}$ being the compactly supported extension of the normal to $\Gamma$, such that $|\vec{n}| \equiv 1$ in a neighborhood of $\Gamma$, as was previously introduced, it is sufficient to show that

$$
\begin{aligned}
& \vec{n} \wedge \vec{u} \in \mathbf{H}^{s+1}(\Omega), \\
& (\vec{u}, \vec{n}) \in H^{s+1}(\Omega) .
\end{aligned}
$$

We shall prove this for $0<s \leqslant 1$. The rest of the proof is easily obtained in the same way, starting from this particular case.

As a consequence of (1.13) and (1.14), we remark that

$$
\left\{\begin{array}{l}
\vec{n} \wedge \vec{u} \in \mathbf{H}^{1}(\Omega) \\
\Delta(\vec{n} \wedge \vec{u})=\vec{n} \wedge \Delta \vec{u}+\vec{f} \\
\vec{n} \wedge \vec{u}=\vec{n} \wedge \Pi \vec{u} \in \mathbf{H}^{s+1 / 2}(\Gamma)
\end{array}\right.
$$

where $\vec{f}$ is a function containing only partial derivatives of $\vec{u}$ of order less than or equal to 1 multiplied by functions in $\mathscr{D}\left(\mathbf{R}^{3}\right)$. It then follows that $\Delta(\vec{n} \wedge \vec{u})$ is in $\mathbf{L}^{2}(\Omega)$. The standard regularity results for elliptic problems (cf. e.g. [10]) then give (1.17).

In the same way, we have

$$
\left\{\begin{array}{l}
(\vec{u}, \vec{n}) \in H^{1}(\Omega), \\
\Delta(\vec{u}, \vec{n}) \in \mathbf{L}^{2}(\Omega),
\end{array}\right.
$$

$\gamma_{1}\{(\vec{u}, \vec{n})\}$ may then be defined in $H^{-1 / 2}(\Gamma)$ (cf. [10]).

In a previous work (cf. [1]), we have established the relation

$$
\gamma_{0} \operatorname{div} \vec{u}=\operatorname{div}_{\Gamma}(\Pi \vec{u})+2 H(\vec{u}, \vec{n})+\gamma_{1}\{(u, n)\},
$$

which holds if $\vec{u}$ is in $\{\mathscr{D}(\bar{\Omega})\}^{3}$ and can be extended by density to those $\vec{u}$ in $\mathbf{H}_{\text {loc }}^{1}(\Delta, \bar{\Omega})$. We have written $H(x)$ for the mean curvature of $\Gamma$ at the point $x$ (i.e. the arithmetic mean of the eigenvalues of the curvature tensor). Thanks to (1.15) and (1.17), we have

$$
\gamma_{1}\{(\vec{u}, \vec{n})\} \in H^{s-1 / 2}(\Gamma)
$$

The classical results on elliptic problems then again lead to (1.18).

2. The Integral Equation. In this section, we introduce the framework in which the variational equation $(0.10)$ can be studied.

2.1. Some Preliminary Lemmas. We shall always write $[v]=\left.v\right|_{\text {int }}-\left.v\right|_{\text {ext }}$ for the jump across $\Gamma$ of the function or of the distribution $v$ which is assumed to admit, in some sense, interior and exterior traces on $\Gamma$.

Proposition 2.1. Let a field $\vec{u} \in \mathbf{L}_{\text {loc }}^{2}\left(\mathbf{R}^{3}\right)$ satisfy $\vec{u} \in \mathbf{H}^{1}\left(\Delta, \Omega^{i}\right) \cap \mathbf{H}_{\text {loc }}^{1}\left(\Delta, \bar{\Omega}^{e}\right)$ and $\Delta \vec{u}+k^{2} \vec{u}=0$ in $\Omega^{i} \cup \Omega^{e}$. Then we have: $\left[\gamma_{1} \operatorname{div} \vec{u}\right]$ is well defined in $H^{-3 / 2}(\Gamma)$ and

$$
\left[\gamma_{1} \operatorname{div} \vec{u}\right]=\operatorname{div}_{\Gamma}([\operatorname{curl} \vec{u} \wedge \vec{n}])-k^{2}[(\vec{u}, \vec{n})]
$$


Proof. div $\vec{u}$ belongs to $H^{0}\left(\Delta, \Omega^{i}\right) \cap H_{\text {loc }}^{0}\left(\Delta, \bar{\Omega}^{e}\right)$. Thus, its second trace may be defined in $H^{-3 / 2}(\Gamma)$ (cf. [10]). For $\varphi \in \mathscr{D}\left(\mathbf{R}^{3}\right)$, (1.9) yields

$$
\begin{aligned}
\int_{\Omega^{i} \cup \Omega^{e}}\{(\Delta \vec{u}, & \operatorname{grad} \varphi)+\operatorname{div} \vec{u} \overline{\Delta \varphi}\} d x \\
& =\left\langle[\operatorname{curl} \vec{u} \wedge \vec{n}], \operatorname{grad}_{\Gamma} \varphi\right\rangle_{-1 / 2,1 / 2}+\left\langle\left[\gamma_{0} \operatorname{div} \vec{u}\right], \gamma_{1} \varphi\right\rangle_{-1 / 2,1 / 2} .
\end{aligned}
$$

Now, $\Delta \operatorname{div} \vec{u}+k^{2} \operatorname{div} \vec{u}=0$ in $\Omega^{i} \cup \Omega^{e}$ also. Then Green's formula

$$
\int_{\Omega^{i} \cup \Omega^{e}}\{(\Delta \vec{u}, \operatorname{grad} \varphi)+\bar{\varphi} \Delta(\operatorname{div} \vec{u})\} d x=-k^{2} \int_{\Gamma}[(\vec{u}, \vec{n})] \bar{\varphi} d \gamma
$$

holds in $\mathbf{H}\left(\operatorname{div}, \Omega^{i}\right) \cap \mathbf{H}_{\text {loc }}\left(\operatorname{div}, \bar{\Omega}^{e}\right)$.

Combining (2.2) and (2.3) and using again Green's formula, we obtain

$$
\begin{aligned}
\left\langle\left[\gamma_{1} \operatorname{div} \vec{u}\right], \varphi\right\rangle_{-3 / 2,3 / 2}= & -\left\langle[\operatorname{curl} \vec{u} \wedge \vec{n}], \operatorname{grad}_{\Gamma} \varphi\right\rangle_{-1 / 2,1 / 2} \\
& -k^{2} \int_{\Gamma}[(\vec{u}, \vec{n})] \bar{\varphi} d \gamma
\end{aligned}
$$

which is (2.1) written in the sense of distributions.

Remark 2.2. We have thus obtained that the "conservation law"

$$
\operatorname{div}_{\Gamma}([\operatorname{curl} \vec{u} \wedge \vec{n}])=k^{2}[(\vec{u}, \vec{n})]
$$

is equivalent to $\left[\gamma_{1} \operatorname{div} \vec{u}\right]=0$.

In the sequel, $\vec{e}$ will denote the field in $\mathbf{L}_{\text {loc }}^{2}\left(\mathbf{R}^{3}\right)$ defined almost everywhere by $\left.\vec{e}\right|_{\Omega^{e}}=$ solution of the exterior problem (1.11) and $\left.\vec{e}\right|_{\Omega^{i}}=$ solution of the interior problem (1.12), assuming that $g \equiv 0$ in the following. We write

$$
\begin{aligned}
& \vec{p}=[\operatorname{curl} \vec{e} \wedge \vec{n}] \in T H^{-1 / 2}(\Gamma), \\
& \lambda=-[(\vec{e}, \vec{n})] \in H^{1 / 2}(\Gamma) .
\end{aligned}
$$

Remark 2.3. If $\vec{c}=-\Pi \vec{e}^{-\mathrm{inc}}$, where $\vec{e}^{\mathrm{inc}}$ is the incident electric field, $\vec{p}$ and $\lambda$ are respectively related to the currents $\vec{j}$ and charges $\rho$ considered in the introduction, by $\vec{p}=i \omega \mu \vec{j}$ and $\lambda=\rho / \varepsilon$.

As a corollary of Proposition 2.1 and of the fact that $\operatorname{div} \vec{e} \equiv 0$ (cf. [1]) in $\Omega^{i} \cup \Omega^{e}$, $\vec{p}$ and $\lambda$ are linked by the conservation law

$$
\operatorname{div}_{\Gamma} \vec{p}+k^{2} \lambda=0
$$

If $\vec{c}$ is a smooth field (i.e. $C^{\infty}$ ), Theorem 1.1 yields

$$
\vec{e} \in\left\{C^{\infty}\left(\bar{\Omega}^{i}\right)\right\}^{3} \cap\left\{C^{\infty}\left(\bar{\Omega}^{e}\right)\right\}^{3}
$$

The representation of solutions of the Helmholtz equation satisfying the Sommerfeld radiation condition (cf. e.g. [15], [22]) gives

$$
\begin{aligned}
& \vec{e}(x)=-\operatorname{grad} v(x)+\vec{a}(x), \quad \text { for all } x \text { not on } \Gamma, \\
& v(x)=\int_{\Gamma} G(x, y) \lambda(y) d \gamma(y),
\end{aligned}
$$




$$
\vec{a}(x)=\int_{\Gamma} G(x, y) p(y) d \gamma(y),
$$

where $G(x, y)$ is Green's kernel given in (0.4).

The classical potential relations (cf. e.g. [11]) enable us to write

$$
\vec{c}=-\operatorname{grad}_{\Gamma} v+\Pi \vec{a} .
$$

Thus, if $\vec{q}$ is a smooth tangential field on $\Gamma$, Stokes formula

$$
\int_{\Gamma}\left(\operatorname{grad}_{\Gamma} v, \vec{q}\right) d \gamma+\int_{\Gamma} v \operatorname{div}_{\Gamma} \vec{q} d \gamma=0
$$

and the continuity equation (2.8) show that $\vec{p}$ is a solution of the following variational equation:

$$
\left\{\begin{array}{l}
\text { Find a smooth tangential field } \vec{p} \text { on } \Gamma \text { such that, } \\
\text { for all } \vec{q} \text { of the same type, } \\
\int_{\Gamma \times \Gamma} G(x, y)\left\{-\frac{1}{k^{2}} \operatorname{div}_{\Gamma} \vec{p}(y) \overline{\operatorname{div}_{\Gamma} \vec{q}(x)}+(\vec{p}(y), \vec{q}(x))\right\} d \gamma(y) d \gamma(x) \\
\quad=\int_{\Gamma}(\vec{c}(x), \vec{q}(x)) d \gamma(x) .
\end{array}\right.
$$

Our aim is now to formulate (2.14) in a framework adapted to the analysis developed subsequently. We set

$$
\begin{aligned}
H & =T H^{-1 / 2}(\Gamma), \\
X & =\left\{\vec{p} \in H: \operatorname{div}_{\Gamma} \vec{p} \in H^{-1 / 2}(\Gamma)\right\}, \\
M & =\left\{\mu \in H^{-1 / 2}(\Gamma) ;\langle\mu, 1\rangle=0\right\} .
\end{aligned}
$$

All these spaces, equipped with their natural norms, are complex Hilbert spaces. It is easily seen, by standard techniques, that the infinitely differentiable elements constitute dense subspaces and that, for any $\vec{p} \in X, \operatorname{div}_{\Gamma} \vec{p} \in M$.

If $X$ and $Y$ are two Banach spaces, $\mathscr{L}(X, Y)$ denotes the Banach space of all bounded linear operators from $X$ into $Y$.

LEMMA 2.4. The operator $\tilde{A}$ defined for infinitely differentiable $\vec{p} \in H$ by

$$
\tilde{A} \vec{p}(x)=\Pi \int_{\Gamma} G(x, y) \vec{p}(y) d \gamma(y)
$$

may be extended by continuity to an operator belonging to $\mathscr{L}\left(H, H^{\prime}\right)$. The problem

$$
\left\{\begin{array}{l}
\text { Find } \vec{a} \in \mathbf{H}_{\mathrm{loc}}^{1}\left(\mathbf{R}^{3}\right) \text { such that } \\
-\left(\Delta \vec{a}+k^{2} \vec{a}\right)=\{\vec{p}\} \quad \text { in } \mathscr{D}^{\prime}(\Omega), \\
\operatorname{curl} \vec{a} \wedge \vec{r} / r+(\vec{r} / r) \operatorname{div} \vec{a}-i k \vec{a}=o(1 / r),
\end{array}\right.
$$

has one and only one solution. We have denoted by $\{\vec{p}\}$ the distribution defined by

$$
\langle\{\vec{p}\}, \varphi\rangle_{\mathscr{D}^{\prime}, \mathscr{D}}=\langle\vec{p}, \Pi \vec{\varphi}\rangle_{H, H^{\prime}} \text { for any } \vec{\varphi} \in\left\{\mathscr{D}\left(\mathbf{R}^{3}\right)\right\}^{3} .
$$

This solution leads to

$$
[\operatorname{curl} \vec{a} \wedge \vec{n}]=\vec{p},
$$




$$
\tilde{A} \vec{p}=\Pi \vec{a} \quad \text { in } H^{\prime} .
$$

Moreover, $\tilde{A}$ can be split into the sum

$$
\tilde{A} \vec{p}=A \vec{p}+R \vec{p},
$$

where $A \vec{p}$ is defined for smooth $\vec{p}$ by

$$
\begin{gathered}
A \vec{p}=\Pi \int_{\Gamma} G_{0}(x, y) \vec{p}(y) d \gamma(y), \\
G_{0}(x, y)=\frac{1}{4 \pi|x-y|},
\end{gathered}
$$

and satisfies the Nedelec-Planchard coerciveness inequality [14]

$$
\exists \alpha>0:\langle A \vec{p}, \vec{p}\rangle \geqslant \alpha\|\vec{p}\|_{H}^{2}, \text { for all } \vec{p} \text { in } H,
$$

and $R$ is a bounded linear operator acting from $T H^{s}(\Gamma)$ into $T H^{s+3}(\Gamma)$ for all real $s$ (cf. [7]).

Proof. The standard results on pseudo-differential operators (cf. [7]) allow us to define $\tilde{A}$ as a bounded operator from $T H^{s}(\Gamma)$ into $T H^{s+1}(\Gamma)$ for all real $s$.

The existence and uniqueness of the solution of (2.19) are obtained in the same way as in the scalar case (cf. [7]) by considering the intermediate problem

$$
\left\{\begin{array}{l}
\text { Find } \vec{u} \in\left\{W_{0}^{1}\left(\mathbf{R}^{3}\right)\right\}^{3}, \forall \vec{v} \in\left\{W_{0}^{1}\left(\mathbf{R}^{3}\right)\right\}^{3} \\
\int_{\mathbf{R}^{3}}(\operatorname{curl} \vec{u}, \operatorname{curl} \vec{v})+\operatorname{div} \vec{u} \overline{\operatorname{div} \vec{v}} d x=\langle\vec{p}, \Pi \vec{v}\rangle,
\end{array}\right.
$$

where $W_{0}^{1}\left(\mathbf{R}^{3}\right)$ is the space (cf. [12])

$$
W_{0}^{1}\left(\mathbf{R}^{3}\right)=\left\{v \in L_{\text {loc }}^{2}\left(\mathbf{R}^{3}\right):(1+r)^{-1} v \in L^{2}\left(\mathbf{R}^{3}\right), \operatorname{grad} v \in \mathbf{L}^{2}\left(\mathbf{R}^{3}\right)\right\} .
$$

Moreover, its solution satisfies the variational relation

$$
\int_{\Omega^{e} \cup \Omega^{i}}\{(\Delta \vec{a}, \vec{v})+(\operatorname{curl} \vec{a}, \operatorname{curl} \vec{v})+\operatorname{div} \vec{a} \overline{\operatorname{div} \vec{v}}\} d x=\langle\vec{p}, \Pi \vec{v}\rangle
$$

for all $\vec{v}$ in $\left\{\mathscr{D}\left(\mathbf{R}^{3}\right)\right\}^{3}$.

Green's formula (1.9) yields

$$
\langle[\operatorname{curl} \vec{a} \wedge \vec{n}], \Pi \vec{v}\rangle+\left\langle\left[\gamma_{0} \operatorname{div} \vec{a}\right],(\vec{v}, \vec{n})\right\rangle=\langle\vec{p}, \Pi \vec{v}\rangle .
$$

Then we obtain (2.21) and

$$
\left[\gamma_{0} \operatorname{div} \vec{a}\right]=0 .
$$

The property (2.22) is obtained from the fact that $\tilde{A}$ and the operator associating $\Pi \vec{a}$ to $\vec{p}$ are both bounded from $H$ to $H^{\prime}$ and coincide on the dense subspace formed by smooth fields. The properties (2.23), (2.24) and (2.26) follow immediately from the results known in the scalar case (cf. [7], [12]).

LEMMA 2.5. Let $\tilde{C}$ be the bounded operator from $H^{s}(\Gamma)$ into $H^{s+1}(\Gamma)$ defined for smooth $u$ by

$$
\tilde{C} u(x)=\int_{\Gamma} G(x, y) u(y) d \gamma(y) .
$$


Let $\vec{p} \in X$ and $\vec{a}$ be the vector potential related to $\vec{p}$ by the above lemma (i.e. by (2.19)). Then, in view of (2.31), we have

$$
\gamma_{0} \operatorname{div} \vec{a}=\tilde{C} \operatorname{div}_{\Gamma} \vec{p}
$$

Proof. If we set $w=\operatorname{div} \vec{a}$, the proof of the previous lemma yields

$$
\left\{\begin{array}{l}
w \in L_{\mathrm{loc}}^{2}\left(\mathbf{R}^{3}\right), \\
\Delta w+k^{2} w=0 \text { in } \Omega^{i} \cup \Omega^{e}, \\
{\left[\gamma_{0} w\right]=0 .}
\end{array}\right.
$$

Due to the fact that $\vec{a} \in \mathbf{H}_{\mathrm{loc}}^{1}\left(\mathbf{R}^{3}\right)$, Proposition 2.1 gives

$$
\left[\gamma_{1} w\right]=\operatorname{div}_{\Gamma} \vec{p}
$$

Since $\vec{a}$ satisfies the radiation condition, we can see (cf. [1]) that $w$ is such that

$$
\frac{\partial w}{\partial r}-i k w=o\left(\frac{1}{r}\right) \text {. }
$$

Rellich's lemma (cf. [18]) and the results of [7] then give $w \in H_{\mathrm{loc}}^{1}\left(\mathbf{R}^{3}\right)$ and (2.33).

LEMMA 2.6. The single layer potential

$$
u(x)=\int_{\Gamma} G(x, y) \rho(y) d \gamma(y)
$$

created by a distribution of charges $\rho$ in $H^{1 / 2}(\Gamma)$ defines an operator acting from $H^{1 / 2}(\Gamma)$ into $T H^{1 / 2}(\Gamma)$ by

$$
\begin{cases}\vec{g}(x)=\operatorname{grad} u(x), & x \text { not lying on } \Gamma, \\ \Pi \vec{g}=\operatorname{grad}_{\Gamma}\left(\gamma_{0} u\right) & \text { on } \Gamma .\end{cases}
$$

Proof. Standard results in potential theory (cf. e.g. [7]) give $u \in H^{2}\left(\Omega^{i}\right) \cap H_{\text {loc }}^{2}\left(\bar{\Omega}^{e}\right)$. Thus, $\vec{g}$ is in $\mathbf{H}^{1}\left(\Omega^{i}\right) \cap \mathbf{H}_{\mathrm{loc}}^{1}\left(\bar{\Omega}^{e}\right)$. The property (2.38) follows since the tangential components of $\vec{g}$ on $\Gamma$ are related only to tangential derivatives of $u$.

LEMMA 2.7. Let a continuous sesquilinear form on $H^{-1 / 2}(\Gamma) \times X$ be defined by

$$
\tilde{b}(\rho, \vec{q})=\left\langle\tilde{C} \rho, \operatorname{div}_{\Gamma} \vec{q}\right\rangle .
$$

Then, $\rho$ belongs to $H^{1 / 2}(\Gamma)$ if and only if there exists $\vec{f} \in T H^{1 / 2}(\Gamma)$ such that

$$
\tilde{b}(\rho, \vec{q})+\langle\vec{f}, \vec{q}\rangle=0 \text { for all } \vec{q} \in X .
$$

Moreover, in this case, we have

$$
\vec{f}=\operatorname{grad}_{\Gamma}\left(\gamma_{0} u\right),
$$

where $u$ is defined by (2.37).

Proof. If $\rho \in H^{1 / 2}(\Gamma)$, then $\gamma_{0} u \in H^{3 / 2}(\Gamma)$. Thus, (2.40) is only the definition of $\operatorname{grad}_{\Gamma}\left(\gamma_{0} u\right)$ in the sense of distributions. Conversely, suppose that (2.40) is satisfied. It follows that $\operatorname{grad}_{\Gamma}\left(\gamma_{0} u\right) \in T H^{1 / 2}(\Gamma)$ with $\gamma_{0} u \in H^{1 / 2}(\Gamma)$. Hence, the properties of the Sobolev spaces (cf. [10]) yield $\gamma_{0} u \in H^{3 / 2}(\Gamma)$. Standard regularity results on elliptic problems then give $\rho \in H^{1 / 2}(\Gamma)$. 
Remark 2.8. All the results given in the previous lemmas $(2.5,2.6,2.7)$, can be transposed to the case where $k=0$ (i.e. for the kernel $G_{0}(x, y)$ defined in (2.25)). In the sequel, we shall use these transpositions and give only essential features which differ from the case where $k>0$.

2.2. The Variational Equation. We can now give a precise formulation of the variational equation (2.14). Although this formulation is used to solve the problem numerically, the numerical analysis of the method cannot be based on it because, roughly speaking, $X$ is not compactly embedded in $H$. That is the reason why we choose the following strategy. We use the fact that the operator $C$, defined for $u \in C^{\infty}(\Gamma)$ by

$$
C u(x)=\int_{\Gamma} G_{0}(x, y) u(y) d \gamma(y)
$$

may be extended by continuity to an isomorphism from $H^{-1 / 2}(\Gamma)$ on $H^{1 / 2}(\Gamma)$. Moreover, it satisfies the coerciveness estimate

$$
\langle C u, u\rangle \geqslant \alpha\|u\|_{-1 / 2, \Gamma,}^{2} \text { for all } u \in H^{-1 / 2}(\Gamma) .
$$

It follows that $C$ is $M$-elliptic and that the "conservation law" (2.8) may be equivalently written in the form

$$
\left\langle C \nu, \operatorname{div}_{\Gamma} \vec{p}+k^{2} \lambda\right\rangle=0 ; \quad \forall \nu \in M .
$$

We introduce the following bounded sesquilinear forms:

$$
\begin{array}{ll}
a(\vec{p}, \vec{q})=\langle A \vec{p}, \vec{q}\rangle ; \quad \vec{p}, \vec{q} \in H ; & \\
r(\vec{p}, \vec{q})=\langle R \vec{p}, \vec{q}\rangle ; \quad \vec{p}, \vec{q} \in H ; & \\
b(\nu, \vec{q})=\left\langle C \nu, \operatorname{div}_{\Gamma} \vec{q}\right\rangle ; \quad \nu \in M, \vec{q} \in X ; \\
s(\nu, \vec{q})=\left\langle S \nu, \operatorname{div}_{\Gamma} \vec{q}\right\rangle ; \quad \nu \in M, \vec{q} \in X ; S=\tilde{C}-C ; \\
c(\mu, \nu)=k^{2}\langle C \mu, \nu\rangle ; \quad \mu \in M ; \nu \in M .
\end{array}
$$

As for $R$ (cf. [7]), $S$ operates from $H^{s}(\Gamma)$ into $H^{s+3}(\Gamma)$ for all real $s$. This improved regularity result $(s+3$ instead of $s+2)$ has not played a great role in the scalar case (see [7]). It will be essential here.

TheOREM 2.9. Let $\vec{c}$ be given in $H^{\prime}=T H^{1 / 2}(\Gamma)$; then $(\vec{p}, \lambda)$ defined by (2.6) and (2.7), is a solution of the variational equation

$$
\left\{\begin{array}{l}
\text { Find }(\vec{p}, \lambda) \in X \times M \text { such that } \\
\tilde{a}(\vec{p}, \vec{q})+\tilde{b}(\lambda, \vec{q})=\langle\vec{c}, \vec{q}\rangle \quad \text { for all } \vec{q} \in X, \\
\frac{b(\nu, \vec{q})}{c}+c(\lambda, \nu)=0 \quad \text { for all } \nu \in M
\end{array}\right.
$$

Conversely, if $(\vec{p}, \lambda)$ is a solution of the variational equation (2.50), then $\lambda \in H^{1 / 2}(\Gamma)$ and the field $\vec{e}$ defined in $\Omega^{i} \cup \Omega^{e}$ by

$$
\vec{e}=-\operatorname{grad} v+\vec{a}
$$

where $\vec{a}$ and $v$ are respectively the scalar and the vector potentials, related to $\lambda$ and $\vec{p}$ by (2.32) and (2.18), is a solution of the exterior problem (1.11) and the interior problem 
(1.12) (with $g=0$ ). In particular, since $k^{2}$ is not an eigenvalue of the interior problem, problem (2.50) has at most one solution.

Proof. Let $(\vec{p}, \lambda)$ be defined by (2.6) and (2.7). From (2.8), $\lambda \in M$ and $\vec{p} \in X$. Consider now a sequence of smooth tangential fields $\vec{c}_{n}$ of $\Gamma$ converging to $\vec{c}$ in $\vec{H}$. By the regularity results of Theorem 1.1 , the related $\left(\vec{p}_{n}, \lambda_{n}\right)$ are smooth and satisfy (2.50) which is another way of writing (2.14). By a continuity argument, $(\vec{p}, \lambda)$ is then a solution of $(2.50)$.

Conversely, if $(\vec{p}, \lambda)$ is a solution of (2.50), Lemma 2.7 gives $\lambda \in H^{1 / 2}(\Gamma)$. Let $\vec{e}$ be defined by (2.51). It is an easy consequence of Lemmas $2.4,2.5,2.6,2.7$, that $\vec{e}$ is a solution of the exterior problem (1.11) and of the interior problem (1.12). If $\vec{c}=0$, then $\left.\vec{e}\right|_{\Omega^{e}}=0$ and $\left.\vec{e}\right|_{\Omega^{i}}$ is a solution of the homogeneous interior problem. The hypothesis assuming that $k^{2}$ is not an eigenvalue of the interior problem yields $\vec{p}=0$ and $\lambda=0$.

3. Fredholm Alternative for a Mixed Formulation. In the previous paragraph, we obtained the existence and uniqueness of the solution of the variational problem (2.50). But this will not be sufficient for the analysis we have in mind. In particular, we need some coercivity estimates which will be established now. The method proposed here will be easily adaptable for other problems of the same kind. In particular, our results can be applied to the mixed formulation of the Helmholtz equation in a bounded open set of $\mathbf{R}^{2}$ with Dirichlet conditions (see [6]).

3.1. The Saddle Point Problem and Brezzi's Conditions. Let us define

$$
L=\left\{\lambda \in H^{1 / 2}(\Gamma): \lambda \in M\right\}
$$

Endowed with the $H^{1 / 2}$ norm, $L$ is a closed subspace of $H^{1 / 2}(\Gamma)$. We then have

THEOREM 3.1. Let $(\vec{c}, \chi)$ be given in $H^{\prime} \times M^{\prime}$. The variational problem

$$
\left\{\begin{array}{l}
\text { Find }(\vec{p}, \lambda) \in X \times M \text { such that } \\
a(\vec{p}, \vec{q})+b(\lambda, \vec{q})=\langle\vec{c}, \vec{q}\rangle \quad \text { for all } \vec{q} \in X \\
\frac{b(\nu, \vec{p})}{b(\chi, \nu\rangle} \text { for all } \nu \in M
\end{array}\right.
$$

has one and only one solution. Moreover, this solution is such that $\lambda \in L$, and if we set $\Lambda(\vec{p}, \lambda)=(\vec{c}, \chi)$ we define an (algebraic and topological) isomorphism from $X \times L$ onto $H^{\prime} \times M^{\prime}$.

The proof will be a consequence of the following lemma:

LEMMA 3.2 ("inf-sup condition"): There exists a constant $\beta>0$ such that

$$
\sup _{\vec{q} \in X}\left\{\frac{1}{\|\vec{q}\|_{X}}|b(\lambda, \vec{q})|\right\} \geqslant \beta\|\lambda\|_{M} \quad \text { for all } \lambda \in M
$$

(In the sequel, it will be understood that a vector is not the zero vector whenever we divide by its norm.) 
Proof. Let $\lambda$ be fixed in $M$. We define $w$ in $H^{1 / 2}(\Gamma)$ via the solution of the problem (cf. [12]):

$$
\begin{aligned}
& \left\{\begin{array}{l}
\text { Find } \tilde{w} \in W_{0}^{1}\left(\mathbf{R}^{3}\right) \text { such that } \\
\int_{\mathbf{R}^{3}}(\operatorname{grad} \tilde{w}, \operatorname{grad} v) d x=\left\langle\lambda, \gamma_{0} v\right\rangle \text { for all } v \in W_{0}^{1}\left(\mathbf{R}^{3}\right),
\end{array}\right. \\
& w=\gamma_{0} \tilde{w} .
\end{aligned}
$$

$\Omega^{e}$ being connected, from a previous result (cf. [1]) we know that the interior problem

$$
\left\{\begin{array}{l}
\text { Find } \vec{u} \in V\left(\Omega^{i}\right) \text { such that } \\
\int_{\Omega^{i}}\{(\operatorname{curl} \vec{u}, \operatorname{curl} \vec{v})+\operatorname{div} \vec{u} \overline{\operatorname{div} \vec{v}}\} d x=\int_{\Gamma} w(\vec{n}, \vec{v}) d \gamma \quad \text { for all } \vec{v} \in V\left(\Omega^{i}\right)
\end{array}\right.
$$

has one and only one solution. We set

$$
V\left(\Omega^{i}\right)=\left\{\vec{u} \in \mathbf{H}^{1}\left(\Omega^{i}\right) ; \vec{n} \wedge \vec{v}=0 \text { on } \Gamma\right\} .
$$

Our aim is now to define $\vec{u}$ in the exterior of $\Omega^{i}$ by solving a problem similar to (3.5). But this needs some preparation. Let us write

$$
\tilde{V}\left(\Omega^{e}\right)=\left\{\vec{u} \in\left\{W_{0}^{1}\left(\Omega^{e}\right)\right\}^{3} ; \vec{n} \wedge \vec{v}=0 \text { on } \Gamma\right\} .
$$

From [1], we can also deduce that there exists a constant $C$ such that

$$
\|\vec{v}\| \omega_{0}^{1}\left(\Omega^{e}\right) \leqslant C\left\{\|\operatorname{curl} \vec{v}\|_{0, \Omega^{e}}+\|\operatorname{div} \vec{v}\|_{0, \Omega^{e}}+\|\vec{v}\|_{0, \Gamma}\right\},
$$

for all $\vec{v}$ in $\tilde{V}\left(\Omega^{e}\right)$, and if $\vec{v} \in \tilde{V}\left(\Omega^{e}\right)$ satisfies

$$
\begin{array}{ll}
\operatorname{curl} \vec{u}=0 & \text { in } \Omega^{e}, \\
\operatorname{div} \vec{u}=0 & \text { in } \Omega^{e},
\end{array}
$$

then there exists $\varphi \in W_{0}^{1}\left(\mathbf{R}^{3}\right)$ satisfying $\varphi \equiv$ constant in $\Omega^{i}$ (here, for simplicity, we have made the nonessential assumption that $\Omega^{i}$ is connected ) such that

$$
\vec{u}=\operatorname{grad} \varphi \quad \text { in } \Omega^{e} .
$$

It follows that $\varphi$ is proportional to the solution $\chi$ of the exterior problem

$$
\left\{\begin{array}{l}
\text { Find } \chi \in W_{0}^{1}\left(\mathbf{R}^{3}\right) \text { such that } \\
\Delta \chi=0 \text { in } \Omega^{e} \cup \Omega^{i} \\
\chi=1 \quad \text { on } \Gamma .
\end{array}\right.
$$

By (3.8)-(3.12) and since the trace operator defines a compact operator from $W_{0}^{1}\left(\Omega^{e}\right)$ into $L^{2}(\Gamma)$, the Peetre lemma (see [1] or a close version in [10]) implies that there exists a constant $C$ such that

$$
\|\vec{v}\|_{W_{0}^{1}\left(\Omega^{e}\right)} \leqslant C\left\{\|\operatorname{curl} \vec{v}\|_{0, \Omega^{e}}+\|\operatorname{div} \vec{v}\|_{0, \Omega^{e}}\right\}
$$

for all $\vec{v}$ in $V\left(\Omega^{e}\right)$, where

$$
V\left(\Omega^{e}\right)=\left\{\vec{v} \in \tilde{V}\left(\Omega^{e}\right) ; \int_{\Gamma}(\vec{n}, \vec{v}) d \gamma=0\right\} .
$$


We have used the fact that the only element in $V\left(\Omega^{e}\right)$ satisfying (3.9) and (3.10) is the zero vector.

Thus, the Lax-Milgram theorem insures that the problem

$$
\left\{\begin{array}{l}
\text { Find } \vec{u} \text { in } V\left(\Omega^{e}\right) \text { such that } \\
\int_{\Omega^{e}}\{(\operatorname{curl} \vec{u}, \operatorname{curl} \vec{v})+\operatorname{div} \vec{u} \overline{\operatorname{div} \vec{v}}\} d x=\int_{\Gamma} w(\vec{n}, \vec{v}) d \gamma \quad \text { for all } \vec{v} \in V\left(\Omega^{e}\right),
\end{array}\right.
$$

has one and only one solution.

Since

$$
\int_{\Gamma} w \frac{\overline{\partial \chi}}{\partial \vec{n}} d \gamma=-\int_{\mathbf{R}^{3}}(\operatorname{grad} w, \operatorname{grad} \chi) d x=-\langle\lambda, \chi\rangle, \quad \gamma_{0} \chi \equiv 1, \lambda \in M,
$$

it follows that we can take $\vec{v} \in \tilde{V}\left(\Omega^{e}\right)$ in (3.15). Thus we can define $\vec{u} \in \mathbf{L}_{\text {loc }}^{2}\left(\mathbf{R}^{3}\right)$ by: $\left.\vec{u}\right|_{\Omega^{i}}$ is the solution of (3.5) and $\left.\vec{u}\right|_{\Omega^{e}}$ is the solution of (3.15). As $\Delta \operatorname{div} \vec{u}=0$ in $\Omega^{e} \cup \Omega^{i},\left[\gamma_{0} \operatorname{div} \vec{u}\right]$ and $\left[\gamma_{1} \operatorname{div} \vec{u}\right]$ can be defined in $H^{-1 / 2}(\Gamma)$ and $H^{-3 / 2}(\Gamma)$, respectively (cf. [10]). From the definition of $\vec{u}$ and Green's formula (1.9), we deduce $\left[\gamma_{0} \operatorname{div} \vec{u}\right]=0$ and $\gamma_{0} \operatorname{div} \vec{u}=w$. Proposition 2.1 gives $\left[\gamma_{1} \operatorname{div} \vec{u}\right]=\operatorname{div}_{\Gamma} \vec{q}$ with $\vec{q}$ defined by

$$
\vec{q}=[\operatorname{curl} \vec{u} \wedge \vec{n}] \in T H^{-1 / 2}(\Gamma) .
$$

From the uniqueness of a single layer potential, it follows that $\vec{q} \in X$ and

$$
\operatorname{div}_{\Gamma} \vec{q}=\lambda \text {. }
$$

The Nedelec-Planchard coerciveness result (2.43) shows again that there exists $\alpha>0$ such that

$$
\left\langle C \lambda, \operatorname{div}_{\Gamma} \vec{q}\right\rangle \geqslant \alpha\|\lambda\|_{M}^{2} .
$$

It is clear that $\vec{q}$ depends continuously on $\lambda$. It follows that there exists a constant $\beta>0$, independent of $\lambda$, and such that

$$
b(\lambda, \vec{q}) \geqslant \beta\|\lambda\|_{M}\|\vec{q}\|_{X} .
$$

This completes the proof of the lemma.

Remark 3.3. As a consequence of the above proof, we obtain that $\vec{u}$ satisfies

$$
\left\{\begin{array}{l}
\Delta \vec{u}=0 \quad \text { in } \Omega^{e} \cup \Omega^{i} \\
\vec{n} \wedge \vec{u}=0 \quad \text { on } \Gamma \\
\gamma_{0} \operatorname{div} \vec{u}=w \in H^{1 / 2}(\Gamma) .
\end{array}\right.
$$

The regularity result given in Theorem 1.1 insures that $\vec{u} \in \mathbf{H}^{2}\left(\Omega^{i}\right) \cap \mathbf{H}_{\text {loc }}^{2}\left(\bar{\Omega}^{e}\right)$ and, then, that $\vec{q} \in T H^{1 / 2}(\Gamma)$. Thus, the previous proof indicates a way to associate $\vec{q} \in T H^{1 / 2}(\Gamma)$ with $\vec{p} \in X$ such that $\operatorname{div}_{\Gamma} \vec{q}=\operatorname{div}_{\Gamma} \vec{p}, \vec{q}$ depending continuously on $\vec{p}$. This property will be fundamental for the numerical analysis which follows.

$M^{\prime}$ is the quotient space $H^{1 / 2}(\Gamma) / \mathrm{C}$. Clearly, for $w \in M^{\prime}$, there is no trouble to say that $w \in H^{1 / 2}(\Gamma), t \geqslant \frac{1}{2}$.

Proof of Theorem 3.1. First, it is clear that $\Lambda \in \mathscr{L}\left(X \times L, H^{\prime} \times M^{\prime}\right)$ is given by

$$
\Lambda(\vec{p}, \lambda)=\left(A \vec{p}-\operatorname{grad}_{\Gamma} C \lambda, C \operatorname{div}_{\Gamma} \vec{p}\right) .
$$


Since $\Lambda$ is continuous, the Banach theorem insures that it will be a topological isomorphism if it is only an algebraic one.

We set

$$
V=\{\vec{p} \in X: b(\nu, \vec{p})=0 \forall \nu \in M\} .
$$

From the coerciveness inequality (2.43), it is clear that $V$ may be equivalently defined by

$$
V=\left\{\vec{p} \in X ; \operatorname{div}_{\Gamma} \vec{p}=0\right\} .
$$

It follows that $V$ is a closed subspace of $X$ and

$$
\|\vec{p}\|_{X}=\|\vec{p}\|_{H} \quad \text { for all } \vec{p} \in V .
$$

This property together with the coerciveness estimate (2.26) for the sesquilinear form $a$ and the "inf-sup condition" (3.2) are the two conditions of Brezzi (cf. [3]) which insure the existence and uniqueness of a solution $(\vec{p}, \lambda)$ to the "saddle-point" problem (3.1). As $\vec{c} \in H^{\prime}$, it follows from Lemma 2.1 that $\lambda \in L$.

We have thus proved that $\Lambda$ is surjective. From the uniqueness of the solution of problem (3.1), it immediately follows that $\Lambda$ is also one-one.

3.2. The Fredholm Alternative.

Corollary 3.4. Let $\Theta \in \mathscr{L}\left(X \times L, H^{\prime} \times M^{\prime}\right)$ be defined by

$$
\Theta(\vec{p}, \lambda)=\left(R \vec{p}-\operatorname{grad}_{\Gamma} S \lambda, k^{2} C \lambda\right) .
$$

\section{Then}

$$
\left\{\begin{array}{l}
\Lambda+\Theta \text { is an (algebraic and topological) isomorphism } \\
\text { from } X \times \text { L onto } H^{\prime} \times M^{\prime} .
\end{array}\right.
$$

Proof. Since $\Theta$ is bounded as an operator from $X \times L$ into $T H^{5 / 2}(\Gamma) \times H^{3 / 2}(\Gamma)$, it is a compact perturbation of the isomorphism $\Lambda$. The operator $\Lambda+\Theta$ is then a Fredholm operator of null index. We obtain (3.26) if we can prove that the first part of the Fredholm alternative holds. This clearly results from the uniqueness of the solution of the variational equation (2.50) (we recall that this is given by the assumption that $k^{2}$ is not an eigenvalue of the interior problem).

Let us give now a coerciveness estimate which will be essential in the numerical analysis we have in mind. Since $\Theta$ may be considered as an operator acting from $H \times M$ into $T H^{3 / 2}(\Gamma) \times M^{\prime}$ (here, the improved order of regularization of $S$ is fundamental), we can introduce a bounded linear operator $T \in \mathscr{L}(H \times M, H \times M)$, defined by

$$
T=I+\Lambda^{-1} \Theta
$$

where $I$ is the identity operator.

THEOREM 3.5. The operator $T$ is an (algebraic and topological) isomorphism from $H \times M$ onto itself. We thus obtain the existence of a constant $\gamma>0$ such that

$$
\|T(\vec{u}, \mu)\|_{H \times M} \geqslant \gamma\|(\vec{u}, \mu)\|_{H \times M} \text { for all }(\vec{u}, \mu) \in H \times M .
$$

(The product of two Hilbert spaces is endowed with the Hilbertian norm.) 
Proof. Let us first show that $\Lambda^{-1} \Theta$ is a compact operator. To this end, let a sequence $\left\{\left(\vec{c}_{n}, \chi_{n}\right)\right\}_{n \in \mathrm{N}}$ in $H^{\prime} \times M^{\prime}$ be such that

$$
\begin{aligned}
\lim \vec{c}_{n}=\vec{c} & \text { strongly in } H^{\prime}, \\
\lim \chi_{n}=\chi & \text { weakly in } M^{\prime} .
\end{aligned}
$$

For any $n \in \mathbf{N}$, we define $\left(\vec{p}_{n}, \lambda_{n}\right) \in X \times L$ by

$$
\Lambda\left(\vec{p}_{n}, \lambda_{n}\right)=\left(\vec{c}_{n}, \chi_{n}\right)
$$

Theorem (3.1) then insures that

$$
\begin{array}{ll}
\lim \vec{p}_{n}=\vec{p} & \text { weakly in } X, \\
\lim \lambda_{n}=\lambda & \text { weakly in } L,
\end{array}
$$

where $(\vec{p}, \lambda)$ is defined by

$$
\Lambda(\vec{p}, \lambda)=(\vec{c}, \chi)
$$

Since $L$ is compactly embedded in $M$, it follows that

$$
\lim \lambda_{n}=\lambda \text { strongly in } M \text {. }
$$

From the definition (3.1) of the operator $\Lambda$ and the coerciveness estimate (2.26), we have

$$
\alpha\left\|\vec{p}_{n}-\vec{p}\right\|_{H}^{2} \leqslant\left\langle\vec{c}_{n}-\vec{c}, \vec{p}_{n}-\vec{p}\right\rangle-b\left(\lambda_{n}-\lambda, \vec{p}_{n}-\vec{p}\right) .
$$

The strong convergences (3.29) and (3.35) yield

$$
\lim \vec{p}_{n}=\vec{p} \quad \text { strongly in } H \text {. }
$$

Since $T H^{3 / 2}(\Gamma)$ is compactly embedded in $H^{\prime}$, we can then deduce that $\Lambda^{-1} \Theta$ is compact.

$T$ is thus a Fredholm operator of null index. If $(\vec{p}, \lambda) \in H \times M$ is such that

$$
(\vec{p}, \lambda)=-\Lambda^{-1} \Theta(\vec{p}, \lambda),
$$

it must be in $X \times L$ and satisfies

$$
(\Lambda+\Theta)(\vec{p}, \lambda)=0 .
$$

The proof is achieved by Corollary 3.4 , the first part of the Fredholm alternative and the bounded inverse theorem.

Let us give now some regularity properties of the solution of problem (3.1). Those of problem (2.50) are identical and will not be repeated.

TheOREM 3.6. Let $(\vec{p}, \lambda) \in X \times L$ satisfy

$$
\Lambda(\vec{p}, \lambda)=(\vec{c}, \chi),
$$

where, $s$ being a nonnegative real number,

$$
\begin{aligned}
& \vec{c} \in T H^{1 / 2+s}(\Gamma), \\
& \chi \in H^{t}(\Gamma), \quad t=\max \left(\frac{1}{2},-\frac{1}{2}+s\right) .
\end{aligned}
$$

Then,

$$
\begin{aligned}
& \vec{p} \in T H^{-1 / 2+s}(\Gamma), \\
& \lambda \in H^{1 / 2+s}(\Gamma) .
\end{aligned}
$$


Proof. Let $\vec{a}$ and $v$ be respectively the vector and the scalar potential related to $\vec{p}$ and $\lambda$ by the kernel $G_{0}$ given in (2.25). We define the field $\vec{e}$ in $\Omega^{i} \cup \Omega^{e}$ by

$$
\vec{e}=\vec{a}-\operatorname{grad} v \text {. }
$$

From Proposition 2.1 and Lemmas 2.4, 2.5, 2.6, 2.7, $\vec{e}$ satisfies

$$
\begin{cases}\Delta \vec{e}=0 & \text { in } \Omega^{e} \cup \Omega^{i} \\ \Pi \vec{e}=\vec{c} & \text { on } \Gamma, \\ \gamma_{0} \operatorname{div} \vec{e}=\chi & \text { on } \Gamma\end{cases}
$$

The regularity properties (3.43) and (3.44) are then the consequences of Theorem 1.1 in the case $s \geqslant 1$. In the case $s=0$, they follow from the definition of the operator $\Lambda$. The intermediate case $0<s<1$ is given by interpolation theory (cf. [10]).

Final Remark 3.6. We have thus established the groundwork for the forthcoming numerical analysis. In order to avoid hypotheses which need to be introduced a priori, we chose not to give an "abstract" framework for our study. Nevertheless, we think the study can be adapted to other problems. In particular, the case of a mixed formulation of the Helmholtz equation in a bounded plane domain $\Omega$ (cf. [6]) is given by the choice $M=L^{2}(\Omega), H=M^{2}, X=H(\operatorname{div} \Omega), L=H_{0}^{1}(\Omega)$, and besides the usual choice of sesquilinear forms $a$ and $b, c$ is $k^{2}$ times the scalar product of $M$, $r$ and $s$ being zero forms.

Centre de Mathématiques Appliquees

Ecole Polytechnique

91128 Palaiseau Cedex, France

1. A. Bendali, Problème aux Limites Extérieur et Intérieur pour le Système de Maxwell en Régime Harmonique, Rapport Interne no. 50, Centre de Mathématiques Appliquées, Ecole Polytechnique, Palaiseau, 1980.

2. A. Bendali, D. Clair \& J. Tourneur, "Finite element approximation of electromagnetic diffraction by arbitrarily-shaped surfaces," Electron. Lett., v. 28, 1982, pp. 641-642.

3. F. BREZzI, "On the existence, uniqueness and approximation of saddle-point problems arising from Lagrangian multipliers," $R$ A I RO Ser. Rouge, v. 8, No. R-2, 1974, pp. 129-151.

4. M. P. Do Carmo, Differential Geometry of Curves and Surfaces, Prentice-Hall, Englewood Cliffs, N.J., 1976.

5. G. Duvaut \& J. L. Lions, Les Inéquations en Mécanique et en Physique, Dunod, Paris, 1972.

6. G. J. Fix \& R. A. Nicolaides, “An analysis of mixed finite element approximations for periodic acoustic wave propagation," SIAM J. Numer. Anal., v. 17, 1980, pp. 779-786.

7. J. GIRoIRE, Integral Equation Methods for Exterior Problems for the Helmholtz Equation, Rapport Interne no. 40, Centre de Mathématiques Appliquées, Ecole Polytechnique, Palaiseau, 1978.

8. J. Giroire \& J. C. Nedelec, "Numerical solution of an exterior Neumann problem using a double-layer potential," Math. Comp., v. 32, 1978, pp. 973-990.

9. R. F. Harrington, "Characteristic modes for antennas and scatterers," in Numerical and Asymptotic Techniques in Electromagnetics (R. Mittra, ed.), Topics in Applied Physics, Vol. 3, SpringerVerlag, Berlin, Heidelberg, New York, 1975.

10. J. L. Lions \& E. Magenes, Problèmes aux Limites Non Homogènes et Applications, Vol. 1, Dunod, Paris, 1968.

11. C. Muller, Foundations of the Mathematical Theory of Electromagnetic Waves, Springer-Verlag, Berlin, 1969.

12. J. C. NedeleC, Approximation des Équations Intégrales en Mécanique et en Phisique. Cours de l'Ecole d'Eté d'Analyse Numérique EDF-CEA-IRIA, Centre de Mathématiques Appliquées. Ecole Polytechnique, Palaiseau, 1977.

13. J. C. NeDElec, "Computation of eddy currents on a surface in $\mathbf{R}^{3}$ by finite element methods," SIAM J. Numer. Anal., v. 15, 1978, pp. 580-595. 
14. J. C. Nedelec \& J. Planchard, “Une méthode variationnelle d'éléments finis pour la résolution numérique d'un problème extérieur dans $\mathbf{R}^{3}$, RAIRO Ser. Rouge, v. 7, R3, 1973, pp. 105-129.

15. D. J. PoGgio \& E. K. MilleR, "Solutions of three-dimensional scattering problems," in Computer Techniques for Electromagnetics (R. Mittra, ed.), Permagon Press, New York, 1973.

16. S. S. M. RAO, D. R. WILTON \& A. W. GLISSON, "Electromagnetic scattering by surfaces of arbitrary shape," IEEE Trans. Antennas and Propagation, v. AP-30, 1982, pp. 409-418.

17. P. A. Raviart \& J. M. Thomas, A Mixed Finite Element Method for 2 nd Order Elliptic Problems, Lecture Notes in Math., Vol. 606, Springer-Verlag, Berlin and New York, 1977, pp. 292-315.

18. F. ReLLiCH, "Über das asymptotische Verhalten der Lösungen von $\Delta u+\lambda u=0$ in unendlichen Gebieten," Jber. Deutsch. Math. Verein., v. 53, 1943, pp. 57-65.

19. V. H. RUMSEY, "Reaction concept in electromagnetic theory," Phys. Rev., v. 94, 1954, pp. 1483-1491.

20. A. SANKar \& T. C. Tong, "Current computation on complex structures by finite element method," Electron. Lett., v. 11, 1975, pp. 481-482.

21. W. L. Stutzman \& G. A. Thiele, Antenna Theory and Design, Wiley, New York, 1981.

22. J. vAN BLADEL, Electromagnetic Fields, McGraw-Hill, New York, 1964.

23. C. H. Wilcox, Scattering Theory for the d'Alembert Equation in Exterior Domains, Lecture Notes in Math., Vol. 442, Springer-Verlag, Berlin, 1975. 